\title{
Prediction of in vivo genotoxicity of lasiocarpine and riddelliine in rat liver using a combined in vitro-physiologically based kinetic modelling-facilitated reverse dosimetry approach
}

\author{
Lu Chen $^{1}$ (D) Ad Peijnenburg ${ }^{2} \cdot$ Laura de Haan $^{1} \cdot$ Ivonne M. C. M. Rietjens $^{1}$
}

Received: 1 May 2019 / Accepted: 4 July 2019 / Published online: 9 July 2019

(c) The Author(s) 2019

\begin{abstract}
Pyrrolizidine alkaloids (PAs) are naturally occurring genotoxic compounds, and PA-containing plants can pose a risk to humans through contaminated food sources and herbal products. Upon metabolic activation, PAs can form DNA adducts, DNA and protein cross links, chromosomal aberrations, micronuclei, and DNA double-strand breaks. These genotoxic effects may induce gene mutations and play a role in the carcinogenesis of PAs. This study aims to predict in vivo genotoxicity for two well-studied PAs, lasiocarpine and riddelliine, in rat using in vitro genotoxicity data and physiologically based kinetic (PBK) modelling-based reverse dosimetry. The phosphorylation of histone protein $\mathrm{H} 2 \mathrm{AX}$ was used as a quantitative surrogate endpoint for in vitro genotoxicity of lasiocarpine and riddelliine in primary rat hepatocytes and human HepaRG cells. The in vitro concentration-response curves obtained from primary rat hepatocytes were subsequently converted to in vivo doseresponse curves from which points of departure (PoDs) were derived that were compared to available in vivo genotoxicity data. The results showed that the predicted PoDs for lasiocarpine and riddelliine were comparable to in vivo genotoxicity data. It is concluded that this quantitative in vitro-in silico approach provides a method to predict in vivo genotoxicity for the large number of PAs for which in vivo genotoxicity data are lacking by integrating in vitro genotoxicity assays with PBK modelling-facilitated reverse dosimetry.
\end{abstract}

Keywords Physiologically based kinetic (PBK) model $\cdot$ Lasiocarpine $\cdot$ Riddelliine $\cdot$ Genotoxicity $\cdot$ In vitro-in vivo extrapolation

$\begin{array}{ll}\text { Abbreviations } \\ \mathrm{BMD}_{10} & \begin{array}{l}\text { The benchmark dose resulting in 10\% extra } \\ \text { effect }\end{array} \\ \gamma \mathrm{H} 2 \mathrm{AX} & \begin{array}{l}\text { Phosphorylated histone } \mathrm{H} 2 \mathrm{AX} \\ \mathrm{C}_{\max }\end{array} \\ \mathrm{AUC} & \text { Maximum concentration } \\ \text { EFSA } & \text { Area under curve } \\ \text { PAs } & \text { Puropean food safety authority } \\ \end{array}$

Electronic supplementary material The online version of this article (https://doi.org/10.1007/s00204-019-02515-5) contains supplementary material, which is available to authorized users.

Lu Chen

Lu.Chen@wur.nl

1 Division of Toxicology, Wageningen University, Stippeneng 4, 6708 WE Wageningen, The Netherlands

2 Wageningen Food Safety Research, PO Box 230, 6700 AE Wageningen, The Netherlands
PBK model Physiologically based kinetic model PoDs Points of departure

\section{Introduction}

Pyrrolizidine alkaloids (PAs) are secondary metabolites that plants produce against insects (Fu et al. 2004). To date, more than 660 PAs and their $\mathrm{N}$-oxides have been identified from more than 6000 plants, and half of them are hepatotoxic (Fu et al. 2010; Wiedenfeld 2011). PA containing plants are widely distributed around the world, and a number of human and livestock poisoning incidents with high mortality due to (unintentional) contamination by PAs have been reported in different countries (Creeper et al. 1999; Edgar et al. 2014; Hill et al. 1997; Molyneux et al. 2011). Human exposure to PAs mainly results from PA containing foods, such as herbal teas, herbal medicines, milk, and honey (Bodi et al. 2014; EFSA 2016, 2017; Kempf et al. 2010; Mathon et al. 2014; Roeder 2000). PAs can induce acute hepatotoxicity in both 
rat and human, resulting in liver necrosis, hepatomegaly, and veno-occlusive disease (VOD) (Fu et al. 2004, 2007; Lin et al. 2011; Mattocks 1986; Wiedenfeld 2011). Chronic exposure to PAs is of concern because of their genotoxicity and carcinogenicity (EFSA 2011). Especially, 1,2-unsaturated PAs are hepatotoxic and considered to be genotoxic carcinogens, thus posing a potential risk to human health (Mori et al. 1985).

Upon metabolic activation PAs can lead to a variety of genotoxic effects, including formation of DNA adducts, DNA and protein cross links, chromosomal aberrations, micronuclei, and DNA double-strand breaks (DSB) (Allemang et al. 2018; Chou et al. 2003, 2004; Fu et al. 2001, 2004, 2010; Prakash et al. 1999; Uhl et al. 2000; Wang et al. 2005a; Xia et al. 2006, 2013). These genotoxic effects may result in gene mutations and tumour formation (Chen et al. 2010; Hoeijmakers 2009). Recently, the European Food Safety Authority (EFSA) has drawn attention to the fact that more data on the toxicokinetics and genotoxicity of individual PAs would greatly refine their risk assessment (EFSA 2017). The state-of-the-art genotoxicity and carcinogenicity data on PAs are limited, and among the 1,2-unsaturated PAs, lasiocarpine and riddelliine are the PAs most studied in in vivo genotoxicity and carcinogenicity studies. For instance, both PAs have been shown to induce genotoxicity in rat liver upon oral exposure (Chan et al. 1994; Xia et al. 2013; Yan et al. 2002). In addition, they were both tested in oral 2-year carcinogenicity studies and shown to induce liver haemangiosarcomas in rats upon chronic oral exposure (NTP 1978, 2003). In addition, limited in vivo genotoxicity data are available for seneciphylline, senkirkine, monocrotaline, and retrorsine (Candrian et al. 1984; Wang et al. 2005a, b), and results from carcinogenicity bioassays have been reported for monocrotaline, clivorine, petasitenine, senkirkine, and symphytine with only in some of these studies rats being dosed via the oral route (Hirono et al. 1976, 1979; Kuhara et al. 1980; Shumaker et al. 1976). Studies to assess whether exposure to PAs results in genotoxicity and carcinogenicity in humans are not available. Lack of genotoxicity studies for most 1,2-unsaturated PAs, hampers PA risk assessment, while economic and ethical constraints limit the possibilities to perform in vivo genotoxicity and carcinogenicity studies for all relevant PAs, including the 17 PAs identified by EFSA to be relevant for exposure via feed and food (EFSA 2017). Thus, alternative testing strategies for estimating the genotoxicity and carcinogenicity of different PAs are required.

In our previous studies, we have successfully predicted in vivo acute liver toxicity for lasiocarpine and riddelliine in rat using physiologically based kinetic (PBK) modelling-facilitated reverse dosimetry (Chen et al. 2018). In this approach, in vitro concentration-response curves for cytotoxicity of lasiocarpine and riddelliine in primary rat hepatocytes were translated to in vivo dose-response curves for acute liver toxicity in rat using PBK modelling (Chen et al. 2018). The aim of the present study was to investigate whether the developed PBK modelling-facilitated reverse dosimetry approach can also adequately translate in vitro concentration-response curves for genotoxicity of lasiocarpine and riddelliine, to in vivo dose-response curves for genotoxicity of these PAs. To this end, the in vitro concentration-responses curves obtained from in vitro genotoxicity studies with primary rat hepatocytes using the $\gamma \mathrm{H} 2 \mathrm{AX}$ in-cell Western (ICW) assay were translated into in vivo dose-response curves using PBK modelling-facilitated reverse dosimetry. The $\gamma \mathrm{H} 2 \mathrm{AX}$ ICW assay allows to quantify the amount of phosphorylated histone $\mathrm{H} 2 \mathrm{AX}(\gamma \mathrm{H} 2 \mathrm{AX})$, which is known to be associated with DNA damage (Audebert et al. 2010; Khoury et al. 2013). Recently, this assay has been successfully applied to quantify a surrogate endpoint for in vitro genotoxic potencies of a large number of PAs, belonging to different chemical classes, in the human liver cell line HepaRG (Louisse et al. 2019). In the present study, the predicted in vivo dose-response curves were used to determine the points of departure (PoDs) which were compared to available in vivo data for genotoxicity induced by lasiocarpine and riddelliine in rats.

\section{Materials and methods}

\section{Chemicals}

Lasiocarpine (>97\%) was purchased from PhytoLab (PhytoLab GmbH \& Co. KG, Germany) (primary rat hepatocyte studies and HepaRG studies), riddelliine (95.4\%) was kindly provided by the National Toxicology Program (NTP) (U.S. Department of Health and Human Service) (primary rat hepatocyte studies) and from Dr. Tao Chen (U.S. FDA) as a generous gift (HepaRG studies). Corning 96 Well cellbind black microplates were obtained from Sigma-Aldrich (St. Louis, MO, USA). The cryopreserved rat (Sprague-Dawley) hepatocytes, thawing/plating supplement pack, cell maintenance supplement pack, fetal bovine serum (FBS), and Williams E Medium (A1217601) were purchased from ThermoFisher (Naarden, The Netherlands). HepaRG cells were obtained from Biopredic International (Saint-Grégoire, France). William's E medium and glutamax were obtained from Life Technologies (Paisley, UK). Primary antibody 'anti-Phospho Histone $\mathrm{H}_{2} \mathrm{AX}$ ' (ser 139) (20E3) rabbit mAb, and secondary antibody $\mathrm{CF} 770$ goat anti rabbit $\operatorname{IgG}(\mathrm{H}+\mathrm{L})$ were purchased from Cell Signaling Technology (Danvers, MA, USA) and Biotium (Fremont, CA, USA), respectively. Fetal bovine serum (FBS) and human insulin were purchased from PanBiotech (Aidenbach, Germany). Penicillin/Streptomycin was from Capricorn Scientific (Ebsdorfergrund, 
Germany). Phosphate-buffered saline (PBS) was obtained from Lonza BioWhittaker (Walkersville, MD, USA). Dimethyl sulfoxide (DMSO) was obtained from Acros Organics (Geel, Belgium). Acetonitrile (UPLC/MS grade) was obtained from Biosolve (Valkenswaard, The Netherlands). Triton X-100, bovine pancreatic RNase A was purchased from Sigma-Aldrich (St Louis). PhosStop tablet was purchased from Roche Diagnostics (Mannheim, Germany). 16\% Paraformaldehyde (PFA) was purchased from Electron microscopy sciences (Hatfield, USA). Reddot was obtained from Biotium (Fremont) and MaxBlock was purchased from Active Motif (Carlsbad, CA, USA).

\section{Cell culture}

Two types of cells were used for determining in vitro concentration-response curves for genotoxicity, i.e., primary rat hepatocytes and HepaRG cells. Briefly, cryopreserved primary rat hepatocytes were seeded at concentration of $5 \times 10^{5}$ cells/well in black 96 well cellbind plates, and incubated in Williams' Medium E (no phenol red) consisting of hepatocyte plating supplement pack (CM3000, serumcontaining) for $4-6 \mathrm{~h}$ before exposure. Only batches with a cell viability $>90 \%$ were used for experiments. HepaRG cells were grown in growth medium consisting of Williams' medium E, supplemented with $10 \% \mathrm{FBS}, 100 \mathrm{IU} / \mathrm{ml}$ penicillin, $100 \mu \mathrm{g} / \mathrm{ml}$ streptomycin, $1 \%$ (v/v) L-glutamine, $5 \mu \mathrm{g} /$ $\mathrm{ml}$ human insulin, and $50 \mu \mathrm{M}$ hydrocortisone-21-hemisuccinate. The growth medium was renewed every 2-3 days. After 2 weeks, the HepaRG cells were trypsinized and seeded at a concentration of $9 \times 10^{3}$ cells/well in black 96 well cellbind plates, and kept on growth medium for 2 weeks and subsequently for another 2 weeks on differentiation medium consisting of growth medium supplemented with $1.7 \%$ (v/v) DMSO (Aninat et al. 2006). The medium was refreshed every 2-3 days. After 2 weeks culturing in differentiation medium, HepaRG cells were fully differentiated and used for experiments within 4 weeks with refreshing the medium every 2-3 days according to the protocol provided by Biopredic International. Primary rat hepatocytes and HepaRG cell were incubated in a $5 \% \mathrm{CO}_{2}: 95 \%$ airhumidified incubator.

\section{YH2AX assay}

The $\gamma \mathrm{H} 2 \mathrm{AX}$ ICW assay allows to simultaneously generate readouts related to genotoxicity and cytotoxicity (Audebert et al. 2010; Khoury et al. 2013) and was used in the present study to assess the in vitro genotoxicity of noncytotoxic concentrations of lasiocarpine and riddelliine in both primary rat hepatocytes and HepaRG cells. 4-6 h after seeding the rat hepatocytes, the medium was replaced by exposure medium Williams' Medium E no phenol red supplemented with hepatocyte plating supplement pack (CM4000, serum free), containing lasiocarpine (final concentrations: 0 (solvent control), 0.006, 0.012, 0.024, $0.048,0.09,0.195,0.39,0.78,1.56,3.125$, and $6.25 \mu \mathrm{M}$ ) or riddelliine (final concentrations: 0 (solvent control), $0.065,0.13,0.26,0.52,1.04,2.08,4.16,8.32,10$, and $15 \mu \mathrm{M})$. Each concentration was tested in three replicates, and two independent experiments with different batches of primary rat hepatocytes were carried out. The HepaRG cells were placed on growth medium supplemented with $0.5 \%$ DMSO for $24 \mathrm{~h}$ before exposure to the compounds. After $24 \mathrm{~h}$ treatment, the HepaRG cells were treated with exposure medium (growth medium without FBS) containing lasiocarpine [final concentrations: 0 (solvent control), $0.78,1.56,3.13,6.25,12.5,25$, and $50 \mu \mathrm{M}]$ or riddelliine [final concentrations: 0 (solvent control), 1.56, 3.13, 6.25, $12.5,25$, and $50 \mu \mathrm{M}]$ in duplicates with three independent experiments. Lasiocarpine and riddelliine used in the $\gamma \mathrm{H} 2 \mathrm{AX}$ assay were added as 200 times concentrated stock solutions in DMSO, and solvent controls were treated with DMSO (final concentration: $0.5 \%$ ).

The $\gamma \mathrm{H} 2 \mathrm{AX}$ in-cell western (ICW) technique was performed essentially as previously described (Audebert et al. 2010; Khoury et al. 2013). Briefly, primary rat hepatocytes and HepaRG cells were treated with lasiocarpine and riddelliine for $24 \mathrm{~h}$; after $24 \mathrm{~h}$ treatment, the medium was removed and the cells were washed with PBS. After washing, the cells were fixed with 4\% PFA in PBS at room temperature, and subsequently washed with PBS. PFA was neutralized with $20 \mathrm{mM}$ $\mathrm{NH}_{4} \mathrm{Cl}$ and the wells were washed with PBS. Cells were permeabilized with $0.2 \%$ Triton X-100 in PBS and washed with PST buffer (PBS containing 2\% FBS and 0.2\% Triton X-100). Cells were blocked with MAXblock Blocking medium supplemented with phosphatase inhibitor PHOSTOP and RNase A, followed by $2 \mathrm{~h}$ incubation with rabbit monoclonal anti $\gamma \mathrm{H} 2 \mathrm{AX}$ in PST buffer. After three washes with PST, secondary detection was carried out using an infrared fluorescent dye conjugated to goat antibody with an absorption peak at $770 \mathrm{~nm}$ in PST buffer. For DNA labelling, RedDot in PST was used together with the secondary antibody. The RedDot signal is used as a measure for cell number, allowing normalization of the $\gamma \mathrm{H} 2 \mathrm{AX}$-response to cell number. Only concentrations resulting in cytotoxicity lower than $20 \%$ (i.e., cell viability of at least $80 \%$ ) were used for further data evaluation. After $1 \mathrm{~h}$ of incubation and subsequent three washes with PST, RedDot and $\gamma \mathrm{H} 2 \mathrm{AX}$ signals were simultaneously visualized using an Odyssey Infrared Imaging Scanner (LiCor ScienceTec, Les Ulis, France). The quantification of phosphorylated histone $\mathrm{H} 2 \mathrm{AX}(\gamma \mathrm{H} 2 \mathrm{AX})$ is expressed relative to the amount of $\gamma \mathrm{H} 2 \mathrm{AX}$ detected in cells exposed to the vehicle control. Error bars represent SD (standard deviation) of the mean. Statistically significant increases in $\mathrm{H} 2 \mathrm{AX}$ phosphorylation after 
treatment were compared with controls using $t$ test with the Graphpad software.

\section{PBK modelling-based reverse dosimetry}

The PBK modelling-facilitated reverse dosimetry approach to predict in vivo genotoxicity based on in vitro genotoxicity data consisted of the following steps: (1) quantification of in vitro concentration-response curves in the $\gamma \mathrm{H} 2 \mathrm{AX}$ assay for lasiocarpine and riddelliine in primary rat hepatocytes and HepaRG cells; (2) translation of the in vitro concentration-response curves to in vivo dose-response curves for rat using previously developed PBK models (Chen et al. 2018); (3) BMD analysis on the predicted in vivo dose-response curves to derive the points of departure (PoDs); and (4) evaluation of the predicted PoD values with the values obtained from in vivo genotoxicity studies.

The structure, model code, and the parameters for the developed rat PBK models for lasiocarpine and riddelliine, including physiological parameters, tissue:blood partition coefficients and parameters for metabolic clearance, were similar to those published previously (Chen et al. 2018). In the step 2, the possible differences in protein binding due to different protein levels in the medium of the in vitro assay and the in vivo situation should be considered. We previously used RED (rapid equilibrium dialysis) to detect the fraction unbound $\left(f_{\mathrm{ub}}\right)$ of lasiocarpine and riddelliine in rat serum, resulting in $f_{\mathrm{ub}}$ values amounting to 0.64 for lasiocarpine and 0.66 for riddelliine (Chen et al. 2018). Thus, the effect concentrations of lasiocarpine and riddelliine in rat blood $\left(C_{\mathrm{Lc} / \mathrm{Rd}}\right.$, rat blood $)$ applied for reverse dosimetry were corrected by the following equation: $C_{\mathrm{Lc} / \mathrm{Rd} \text {, rat blood }}=C_{\mathrm{ub} \text {, in vitro }} / f_{\mathrm{ub} \text {, rat blood }}$, where $C_{\mathrm{ub}}$, in vitro is the unbound concentration of lasiocarpine and riddelliine in the in vitro medium. Since the exposure medium used in the present study for the in vitro genotoxicity assay was serum free, the concentration of lasiocarpine and riddelliine in the in vitro $\gamma \mathrm{H} 2 \mathrm{AX}$ assay was considered to be equal to the unbound concentration in rat serum.

When considering an endpoint-like genotoxicity, the total dose over time, and thus, the area under the curve (AUC) presents the most relevant parameter for reverse dosimetry (Groothuis et al. 2015). To this end, the in vitro concentration-response data were translated to in vitro AUC-response data, by multiplying the effect concentration with the assay time (24 h) (Daston et al. 2010). The AUC-response curves thus obtained were translated to the in vivo situation setting them equal to the unbound AUC values in the plasma.

\section{BMD analysis of in vitro concentration-response curves and of predicted in vivo dose-response data}

To support the validity and use of the in vitro-in silico approach the predicted $\mathrm{BMD}_{10}$ (the benchmark dose responsible for a $10 \%$ response), values for lasiocarpine and riddelliine were compared with PoDs derived from in vivo genotoxicity data from the literature (Chan et al. 1994; Xia et al. 2013; Yan et al. 2002). The continuous model using summary data from the PROAST software (version 66.42, the Dutch National Institute for Public Health and the Environment, The Netherlands) was applied to analyse the predicted in vivo dose-response data for lasiocarpine and riddelliine and to derive the $\mathrm{BMD}_{10}$ values. The goodness of fit was used to judge if the model could be accepted. Only a model fit with $P<0.05$ was considered acceptable for the determination of $\mathrm{BMD}_{10}$ values.

\section{Results}

\section{In vitro $\mathrm{YH} 2 \mathrm{AX}$ assay}

Figure 1a, b presents the in vitro concentration-response curves obtained for lasiocarpine and riddelliine in the $\gamma \mathrm{H} 2 \mathrm{AX}$ assay, which provides a surrogate endpoint for genotoxicity determined in primary rat hepatocytes and metabolic competent HepaRG cells, respectively. The PROAST program was applied to analyse the concentration-response data and to determine the critical effect concentration, i.e., $\mathrm{BMC}_{10}$ (the benchmark concentration responsible for a $10 \%$ response). Histone $\mathrm{H} 2 \mathrm{AX}$ phosphorylation in these two types of cells increased upon exposure to lasiocarpine and riddelliine in a dose-dependent manner (Fig. 1). Table 1 shows the $\mathrm{BMC}_{10}$ values of lasiocarpine and riddelliine derived from these concentration-response curves. The primary rat hepatocytes appear more sensitive towards the $\gamma \mathrm{H} 2 \mathrm{AX}$ induction-related genotoxicity of lasiocarpine and riddelliine than the HepaRG cells, since the $\mathrm{BMC}_{10}$ values for $\gamma \mathrm{H} 2 \mathrm{AX}$ induction-related genotoxicity for lasiocarpine and riddelliine derived from primary rat hepatocytes were, respectively, 105- and 31-fold lower than those obtained in the HepaRG cells.

\section{Translation of the in vitro concentration-response curves to the in vivo dose-response curves and prediction of in vivo $B M D_{10}$ values}

The in vitro concentration-response curves obtained for primary rat hepatocytes were first corrected for differences in binding to proteins in medium (in vitro) and serum (in vivo), resulting in effect concentrations, which were then converted to AUC-response curves by multiplying the effect concentration with $24 \mathrm{~h}$, the duration of the in vitro incubations. Figure 2 a presents the AUC-response curves thus obtained. These in vitro AUC-response curves were subsequently translated into predicted dose-response curves using the respective PBK models (Fig. 2b). The dose levels 

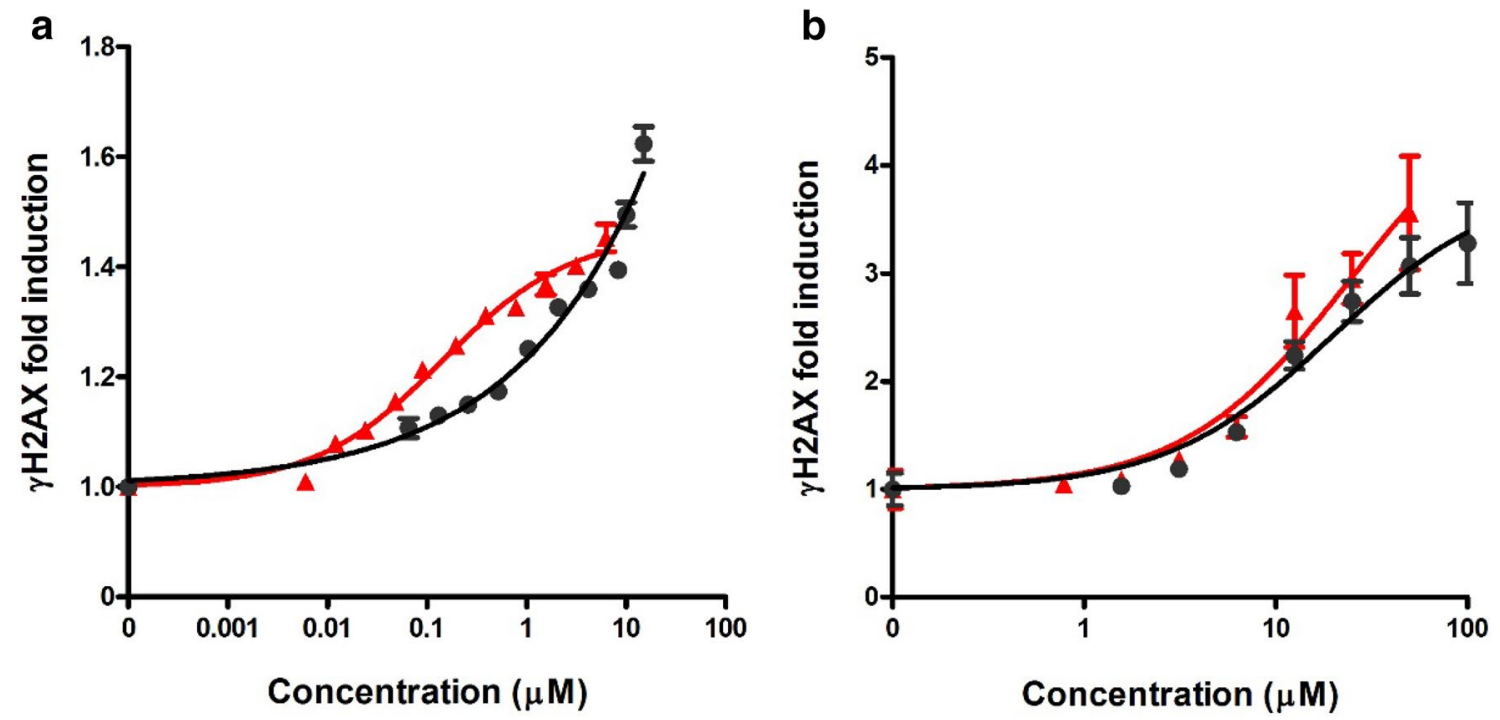

Fig. 1 Concentration-response curves for $\gamma \mathrm{H} 2 \mathrm{AX}$ induction in primary rat hepatocytes (a) and HepaRG cells (b) treated with increasing concentration of lasiocarpine (red triangle) and riddelliine (black circle) for $24 \mathrm{~h}$ (mean $\pm \mathrm{SD}$ ) (color figure online)

Table $1 \mathrm{BMC}_{10}$ values for lasiocarpine and riddelliine derived from the concentration-response curves presented in Fig. 1

\begin{tabular}{ll}
\hline Cell model & $\mathrm{BMC}_{10}(\mu \mathrm{M})$ \\
\hline Primary rat hepatocytes & \\
Lasiocarpine & 0.02 \\
Riddelliine & 0.07 \\
HepaRG cells & \\
Lasiocarpine & 2.09 \\
Riddelliine & 2.17 \\
\hline
\end{tabular}

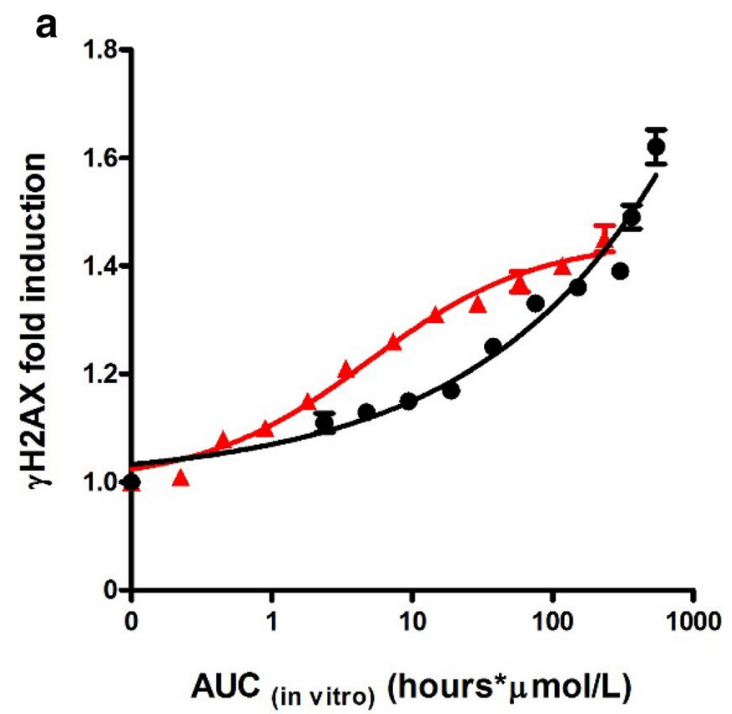

Fig. 2 In vitro AUC-response curves for lasiocarpine and riddelliine obtained with primary rat hepatocytes using the $\gamma \mathrm{H} 2 \mathrm{AX}$ assay (a) and in vivo dose-response curves for genotoxicity in rat liver predicted thus obtained for the dose-response curves are shown in the Supplementary data (Table 1). From these predicted dose-response curves, the $\mathrm{BMD}_{10}$ values for lasiocarpine and riddelliine were derived amounting to 8.82 and $3.41 \mathrm{mg} /$ $\mathrm{kg}$ bw/day, respectively (Table 2). Compared to the in vitro $\gamma \mathrm{H} 2 \mathrm{AX}$ induction-related genotoxicity data in primary rat hepatocytes, where lasiocarpine was 3.5-fold more active than riddelliine (Figs. 1a, 2a), the predicted in vivo genotoxicity of riddelliine appeared to be 2.6-fold higher than that of lasiocarpine (Fig. 2b). This can be ascribed to the differences

b

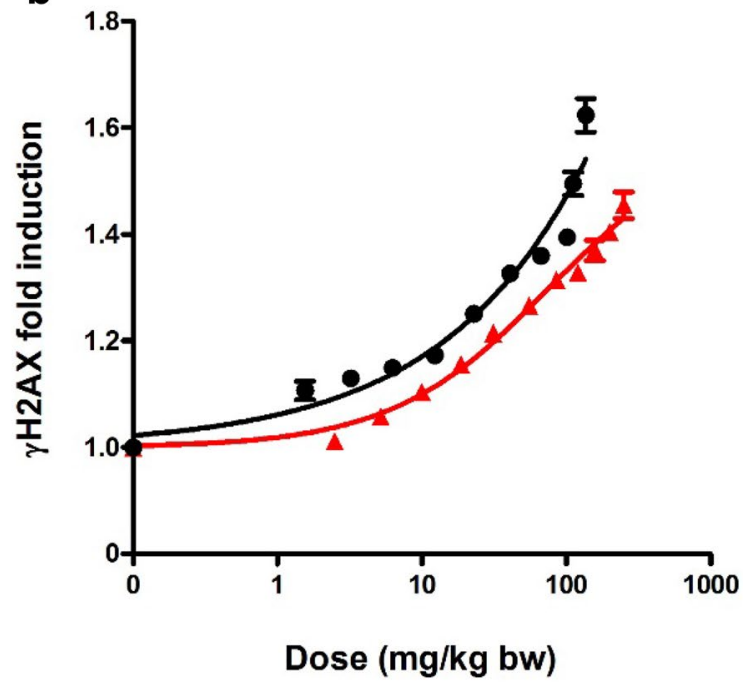

from in vitro AUC-response curves using PBK modelling (b). Lasiocarpine (red triangle); riddelliine (black circle). Data expressed as $($ mean $\pm \mathrm{SD})$ (color figure online) 
Table 2 Predicted $\mathrm{BMD}_{10}$ values for lasiocarpine and riddelliine obtained using PBK modelling-facilitated reverse dosimetry, which allowed translation of the in vitro AUC-genotoxicity curves (Fig. 2a) to the in vivo dose-response curves (Fig. 2b)

\begin{tabular}{ll}
\hline Compound & $\begin{array}{l}\text { Predicted } \\
\mathrm{BMD}_{10}(\mathrm{mg} / \mathrm{kg} \\
\text { bw/day })\end{array}$ \\
\hline Lasiocarpine & 8.82 \\
Riddelliine & 3.41 \\
\hline
\end{tabular}

in kinetics with a slower clearance of riddelliine compared to lasiocarpine, with the total scaled in vivo catalytic efficiency in rat for depletion of riddelliine previously shown to be 11-fold lower than that for lasiocarpine (Chen et al. 2018).

\section{Evaluation of the predicted PoDs for in vivo genotoxicity}

To evaluate the outcomes of the in vitro-PBK model-based predictions for dose-dependent $\gamma \mathrm{H} 2 \mathrm{AX}$ induction-related genotoxicity of lasiocarpine and riddelliine in rat liver, the predicted $\mathrm{BMD}_{10}$ values for lasiocarpine and riddelliine were compared to the in vivo genotoxicity data in rat liver that have been published previously (Chan et al. 1994; Xia et al. 2013; Yan et al. 2002). Table 3 presents an overview of literature data in rat hepatocytes in vivo, measuring endpoints including DNA-adduct formation and unscheduled DNA synthesis. The data from these in vivo animal studies were not suitable for BMD analysis due to the limited number of data points and insufficient distribution of the data points over the dose-response curves. Therefore, the lowest observed adverse effect level (LOAEL) of lasiocarpine and riddelliine that induced in vivo genotoxicity was used for the validation (Table 3). Figure 3a, b shows a comparison of the predicted $\mathrm{BMD}_{10}$ values for in vivo genotoxicity of lasiocarpine and riddelliine and these LOAEL values derived from in vivo literature rat data for genotoxicity of lasiocarpine and riddelliine. For lasiocarpine, the predicted $\mathrm{BMD}_{10}$ was only 1.1-fold different (lower) compared to the LOAEL derived from the in vivo study detecting DNA-adduct formation (Xia et al. 2013) (Fig. 3a), while it was higher than the dose level of $1.85 \mathrm{mg} / \mathrm{kg}$ bw at which lasiocarpine-derived DNA adducts were not yet detectable. At this dose level, riddelliine DNA adducts could already be detected in the in vivo study, which is in line with the predicted $\mathrm{BMD}_{10}$ values which indicated riddelliine to have a lower $\mathrm{BMD}_{10}$ value than lasiocarpine. For riddelliine, the predicted $\mathrm{BMD}_{10}$ was only 1.8-fold different (higher) compared to the LOAEL derived from in vivo experimental data measuring

Table 3 In vivo rat genotoxicity data of lasiocarpine and riddelliine obtained from the literature

\begin{tabular}{|c|c|c|c|c|c|c|c|c|}
\hline Compound & Species & Exposure route & $\begin{array}{l}\text { Dose }(\mathrm{mg} / \mathrm{kg} \\
\text { bw/day) }\end{array}$ & $\begin{array}{l}\text { Type of expo- } \\
\text { sure }\end{array}$ & $\begin{array}{l}\text { Exposure } \\
\text { duration }\end{array}$ & Effect & $\begin{array}{l}\text { The LOAEL } \\
\text { inducing geno- } \\
\text { toxicity (mg/ } \\
\text { kg bw/day) }\end{array}$ & Study \\
\hline Lasiocarpine & Rat & Oral & $0,1.85,9.88$ & Repeated & 3 days & $\begin{array}{c}\text { Level of DNA } \\
\text { adducts: } 0, \\
0,25.2 / 10^{8} \\
\text { nucleotides }\end{array}$ & 9.88 & Xia et al. (2013) \\
\hline Riddelliine & Rat & Oral & $0,1.85,9.88$ & Repeated & 3 days & $\begin{array}{c}\text { Level of DNA } \\
\text { adducts: } 0 \\
3.04 / 10^{8} \\
13.2 / 10^{8} \\
\text { nucleotides }\end{array}$ & 1.85 & Xia et al. (2013) \\
\hline Riddelliine & Rat & Oral & 0,10 & Repeated & 3 days & $\begin{array}{l}\text { Level of DNA } \\
\text { adducts: } 0 \text {, } \\
27 / 10^{7} \text { nucleo- } \\
\text { tides }\end{array}$ & 10 & Yan et al. (2002) \\
\hline Riddelliine & Rat & Oral & $\begin{array}{l}0,0.33,1.0 \\
3.3\end{array}$ & Repeated & 5 days & $\begin{array}{l}\text { Percentage of } \\
\text { hepatocytes } \\
\text { showing } \\
\text { induction } \\
\text { of unsched- } \\
\text { uled DNA } \\
\text { synthesis: } \\
0.66 \pm 0.27 \\
1.98 \pm 0.54 \\
4.22 \pm 0.83^{* *} \\
7.56 \pm 3.50 * *\end{array}$ & 1.0 & $\begin{array}{c}\text { Chan et al. } \\
\text { (1994) }\end{array}$ \\
\hline
\end{tabular}

**Significantly different from the control group $(P \leq 0.01)$ by Dunn's test or Shirley's test (Chan et al. 1994) 


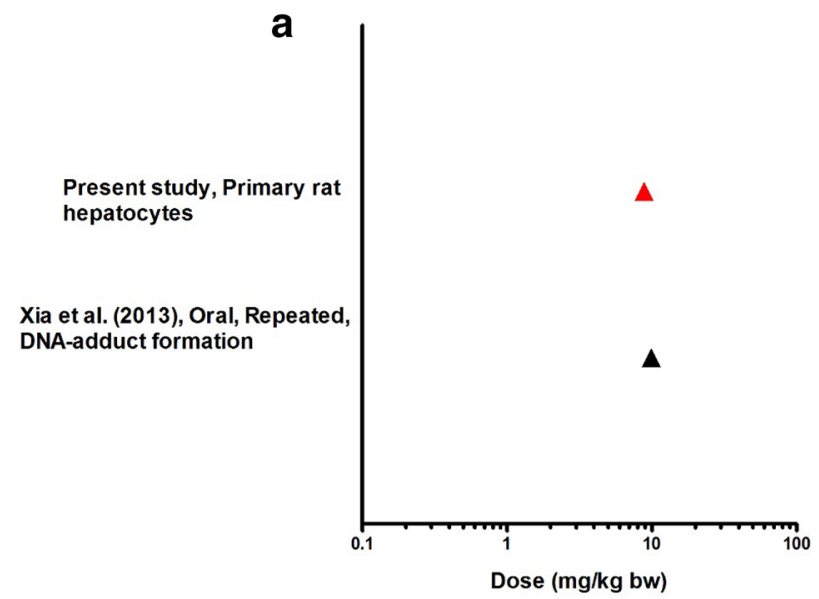

Fig. $3 \mathrm{BMD}_{10}$ values for genotoxicity of (a) lasiocarpine (red triangle) and (b) riddelliine (red circle) predicted by the PBK modellingfacilitated reverse dosimetry approach using data from the $\gamma \mathrm{H} 2 \mathrm{AX}$ assay in primary rat hepatocytes from the present study and as compared to the LOAEL of (a) lasiocarpine derived from an in vivo

DNA-adduct formation (Xia et al. 2013). Compared to the dose level, inducing detectable DNA-adduct formation by riddelliine of $10 \mathrm{mg} / \mathrm{kg}$ bw/day in the study reported by Yan et al. (Yan et al. 2002) showed that the prediction is 2.9-fold lower, but this may in part be due to the fact that the effective dose level reported in this study was the only dose level tested, and dose levels lower than $10 \mathrm{mg} / \mathrm{kg}$ bw/day were not included in the study. Compared to the lowest dose level that causes a statistically significant increase in unscheduled DNA synthesis, the predicted $\mathrm{BMD}_{10}$ for riddelliine was 3.4fold higher (Chan et al. 1994).

\section{Discussion}

The aim of the present study was to assess whether the developed PBK modelling-facilitated reverse dosimetry could adequately predict in vivo dose-response curves for genotoxicity of lasiocarpine and riddelliine in rats. The $\gamma \mathrm{H} 2 \mathrm{AX}$ ICW assay was used to quantify a surrogate endpoint for genotoxicity of these PAs in primary rat hepatocytes. So far, the $\gamma \mathrm{H} 2 \mathrm{AX}$ ICW assay has been successfully applied as a surrogate endpoint for evaluating the genotoxicity of several food contaminants, including aflatoxins (Theumer et al. 2018), heavy metals (Kopp et al. 2018), polycyclic aromatic hydrocarbons (Audebert et al. 2010, 2012), and PAs (Louisse et al. 2019), for possible genotoxicity.

To date, lasiocarpine and riddelliine have been reported to induce genotoxic effects in in vitro assays for different endpoints including formation of micronuclei (Allemang et al. 2018), DNA adducts (Xia et al. 2006), chromosomal aberrations (Takanashi et al. 1980), DNA-cross links (Kim

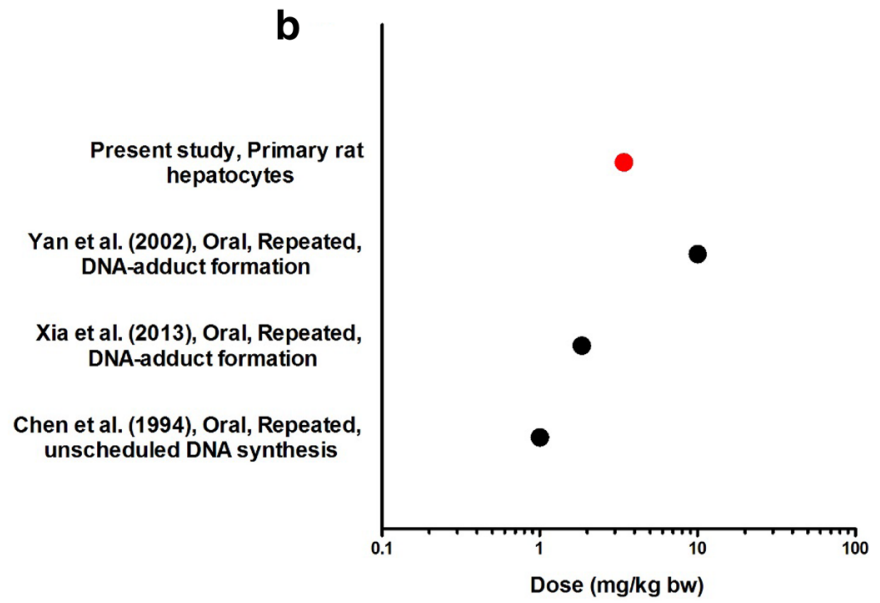

animal study by Xia et al. (2013) reporting DNA-adduct formation (black triangle), and of (b) riddelliine derived from in vivo animal studies (black dots) reporting DNA-adduct formation (Xia et al. 2013; Yan et al. 2002) and unscheduled DNA analysis (Chan et al. 1994) (color figure online)

et al. 1995), and sister chromatid exchange (Chan et al. 1994). $\gamma \mathrm{H} 2 \mathrm{AX}$ occurs after a DNA double-strand break in a cell, and $\gamma \mathrm{H} 2 \mathrm{AX}$ induction has been reported to correlate strongly with DNA damage and is a well-recognized pre-cancerous biomarker in vivo, present in all cell types (Bonner et al. 2008; Singh et al. 2011). The induction of $\gamma \mathrm{H} 2 \mathrm{AX}$ can reflect a global genotoxic insult that may originate from different types of DNA damage: DNA-adduct formation, DNA single-strand breaks, DNA replication, or transcription blocking lesions (Sedelnikova et al. 2010). The $\gamma \mathrm{H} 2 \mathrm{AX}$ ICW assay has been reported to detect genotoxins with such a variety of DNA-damaging characteristics (Khoury et al. 2013). It is also important to note that H2AX phosphorylation could be linked to apoptosis or necrosis rather than to true genotoxicity (Imreh et al. 2011). One of the main advantages of the $\gamma \mathrm{H} 2 \mathrm{AX}$ assay is that it can simultaneously produce readouts that indicate genotoxicity and cytotoxicity (Graillot et al. 2012). To minimize the possible impact of false-positive genotoxic results, e.g., apoptosis or necrosis related $\gamma \mathrm{H} 2 \mathrm{AX}$ induction, the cut-off value for cytotoxicity was set to $80 \%$ viable cells in the present study which is in line with the previous studies (Clarke et al. 2012; O'donovan 2012). Comparison of the results obtained using the $\gamma \mathrm{H} 2 \mathrm{AX}$ assay to published genotoxic data obtained with other in vitro bioassays, including formation of micronuclei (Allemang et al. 2018), DNA adducts (Xia et al. 2006), chromosomal aberrations (Takanashi et al. 1980), DNAcross links (Kim et al. 1995), and sister chromatid exchange (Chan et al. 1994), indicates that the $\gamma \mathrm{H} 2 \mathrm{AX}$ assay provides a relevant surrogate readout for in vitro genotoxicity of PAs.

In the present study, both primary rat hepatocytes and HepaRG cells were used to perform the $\gamma \mathrm{H} 2 \mathrm{AX}$ assay, 
since these two types of cells contain the CYP450 enzymes required for biotransformation of lasiocarpine and riddelliine into DNA reactive intermediates (Fu et al. 2004). HepaRG cells are derived from a human hepatocellular carcinoma, and express most of the CYP450 enzymes, especially the CYP 3A and 2B isoforms, that are key enzymes for PAs bioactivation (Fu et al. 2004; Guillouzo et al. 2007; Lin et al. 2003; Prakash et al. 1999; Turpeinen et al. 2009). It is important to note that the metabolic activation of PAs in rat hepatocytes is different from that in human hepatocytes and human HepaRG cells. The metabolism of PAs in rodents is mainly catalyzed by CYP $2 \mathrm{~B}$ and CYP3A subfamilies, while in humans, metabolism is mainly catalyzed by CYP 3A4 (Li et al. 2011; Miranda et al. 1991). To date, the activity of CYP $3 \mathrm{~A}$ and CYP $2 \mathrm{~B}$ isoforms in primary rat hepatocytes have not been fully quantified, making it difficult to directly compare the activity of these individual enzymes to the activity of CYP3A4 in human hepatocytes and HepaRG cells. However, comparisons of the activity of CYP450s in HepaRG cells and primary human hepatocytes have been reported. The results were somewhat inconsistent across different studies. Gerets et al. (2012) reported that CYP 3A4 activity was about 17.0-fold lower in the HepaRG cells than in primary human hepatocytes from three different donors (Gerets et al. 2012). However, Kvist et al. (2018), and Lübberstedt et al. (2011) who investigated the CYP 1A2, CYP 2B6, CYP 2C8, CYP 2C9, CYP 2C19, and CYP 2D6 activities in HepaRG cells and primary human hepatocytes, showed that these enzyme activities were generally lower in HepaRG cells than those in human hepatocytes, except for CYP 3A4 which showed a generally 1.5 -fold higher activity in HepaRG cells (Kvist et al. 2018; Lübberstedt et al. 2011). We previously investigated the cytotoxicity of lasiocarpine and riddelliine in primary rat hepatocytes, primary human hepatocytes and HepaRG cells, providing an indirect measurement of the CYP-mediated bioactivation of these PAs in the different cell models. The results showed that the IC50 value of lasiocarpine for effects on cell viability in the primary rat hepatocytes was 2- and 20-fold lower than the IC50 values for the primary human hepatocytes and HepaRG cells, respectively; the IC50 value of riddelliine in the primary rat hepatocytes was sevenfold and 22 -fold lower than the values for the primary human hepatocytes and HepaRG cells, respectively (Chen et al. 2018; Ning et al. 2019). Given that CYP 3A4 is the main player in PA bioactivation in the primary human hepatocytes and HepaRG cells ( $\mathrm{Li}$ et al. 2011; Miranda et al. 1991), our data suggest that HepaRG cells may have a lower CYP 3A4 activity compared to primary human hepatocytes, as well as a lower capability in PAs bioactivation compared to primary human hepatocytes and rat hepatocytes. Given that the in vivo data for evaluation of the predictions were from rat, predictions based on data from the $\gamma \mathrm{H} 2 \mathrm{AX}$ assay obtained in rat hepatocytes provide the best basis to make the predictions. Quantifying $\gamma \mathrm{H} 2 \mathrm{AX}$ formation as the endpoint for genotoxicity, the primary rat hepatocytes appeared to be about one-to-two orders of magnitude more sensitive to lasiocarpine and riddelliine induced genotoxicity than the HepaRG cells. Allemang et al. (2018) investigated genotoxicity of lasiocarpine and riddelliine in HepaRG cells using the micronucleus assay, showing dose-dependent increases in micronucleus induction. The $\mathrm{BMC}_{10}$ values for lasiocarpine and riddelliine derived from these concentration-response curves amounted to 0.13 and $0.63 \mu \mathrm{M}$, respectively (Allemang et al. 2018). Comparison of these values to the $\mathrm{BMC}_{10}$ values derived from the HepRG cells in the $\gamma \mathrm{H} 2 \mathrm{AX}$ assay in the present study (Table 1) reveals that the micronucleus endpoint is 16.1and 3.4-fold more sensitive for lasiocarpine and riddelliine, respectively. Compared to the results obtained in the present study using the primary hepatocytes in the $\gamma \mathrm{H} 2 \mathrm{AX}$ assay, the HepaRG cells in the micronucleus assay were still 6.5and 9.0-fold less sensitive for lasiocarpine and riddelliine, respectively. It is concluded that for quantitative in vitro to in vivo extrapolation (QIVIVE), use of primary hepatocytes is favoured over use of HepaRG cells. For this reason, and also because of the availability of rat in vivo data, in the present study, the results obtained in the primary rat hepatocytes were used for the reverse dosimetry.

For this reverse dosimetry, we used the AUC instead of the the maximum concentration $\left(C_{\max }\right)$ as the selected metric. When predicting toxicity for which a threshold exists and thus a concentration below which there will be no effect, $C_{\max }$ may be the selected parameter for the reverse dosimetry, while the use of the AUC would be relevant for endpoints such as genotoxicity or tumour formation that depend more on the total dose than on peak concentrations (Groothuis et al. 2015). In the present study, the predicted PoDs based on the AUC approach were comparable with available in vivo data on genotoxicity of lasiocarpine and riddelliine in rat liver (Fig. 3). When making the prediction based on the $C_{\max }$ approach, the predicted $\mathrm{BMD}_{10}$ of lasiocarpine and riddelliine were up to 17.4- and 42.2-fold lower compared to in vivo animal data. This corroborates that the prediction of genotoxicity for the target compound based on the AUC approach is an adequate choice (Groothuis et al. 2015).

It is of interest to compare the values obtained for in vivo genotoxicity of lasiocarpine and riddelliine to PoDs derived from available tumour data. From data on the incidence of liver, haemangiosarcoma obtained in 2-year carcinogenicity studies in rats upon chronic oral exposure to lasiocarpine and riddelliine (NTP 1978, 2003). EFSA derived a $\mathrm{BMD}_{10}$ of $0.131 \mathrm{mg} / \mathrm{kg}$ bw/day for lasiocarpine and of $0.292 \mathrm{mg} / \mathrm{kg}$ bw/day for riddelliine (EFSA 2017). These $\mathrm{BMD}_{10}$ values obtained from the 2-year carcinogenicity studies are oneto-two orders of magnitude lower than the $\mathrm{BMD}_{10}$ levels 
predicted in the present study and the dose levels used in other studies to detect genotoxicity (Chan et al. 1994; Xia et al. 2013; Yan et al. 2002) (Fig. 3). This may be related to the fact that the exposure duration in the genotoxicity studies is far shorter (1-5 days) than the 2-year exposure applied in carcinogenicity studies.

It is also of interest to consider that in risk assessment for PAs, the estimate daily intake (EDI) values were calculated based on total PA levels in food, taking into consideration combined exposure to different PAs (BfR 2013; EFSA 2016, 2017). When considering combined exposure, different potencies of the various PAs could be considered. This implies that combined exposure could take their relative potency (REP) into account to 'adjust' the individual PA concentrations. The concept of REP factors describes the relative toxic potency of each congener in comparison with a selected reference congener. The most well-established example in toxicology for this approach is the equivalency concept for polychlorinated dioxins, furans, and PCBs (Van den Berg et al. 2006). Similar approaches were suggested for phototoxic furocoumarins (Raquet and Schrenk 2014), or are applied in pharmacology to synthetic glucocorticoids (Suzuki et al. 2015). Recently, Allemang et al. (2018) determined the concentration-dependent PA-induced formation of micronuclei in HepaRG cells by exposing the cells to 15 PAs and calculated the REP values based on these concentration-response curves, and lasiocarpine was found to be six times more potent than riddelliine (Allemang et al. 2018). In the present study, we assessed genotoxicity of lasiocarpine and riddelliine in primary rat hepatocytes based on the $\gamma \mathrm{H} 2 \mathrm{AX}$ assay and found that lasiocarpine was 3.5-fold more potent than riddelliine. These REP values, however, are based on in vitro data and do not take the differences in toxicokinetics into account. The results of the present study, in which the concentration-response curves were translated to in vivo dose-response curves using PBK modelling, reveal that the differences in toxicokinetics between different PAs may be substantial and may influence their ultimate in vivo REP values. On the basis of the predicted in vivo rat genotoxicity, riddelliine was found to be more potent (2.6fold) than lasiocarpine, which is in agreement with in vivo genotoxicity findings. When aiming to extend the approach of the present study to other PAs, the kinetic and metabolic properties of these PAs should be incorporated in the PBK models applied to take into account possible PA-specific kinetic properties. For instance, the translocation rate of heliotrine and especially echimidine across a Caco-2-cell monolayer was shown to be markedly lower compared to the passage rate of senecionine and senkirkine, most likely due to active P-glycoprotein-mediated transport of heliotrine and echimidine from the intestinal cells back to the apical side representing the gut lumen (Hessel et al. 2014). For this example, the ka value for absorption of the PA from the gastrointestinal tract into the liver should be modified to take these absorption characteristics into account.

In conclusion, we demonstrated that the PBK modellingfacilitated reverse dosimetry can adequately translate in vitro concentration-response curves for lasiocarpine and riddelliine, obtained using the $\gamma \mathrm{H} 2 \mathrm{AX}$ assay in primary hepatocytes, to in vivo dose-response curves for genotoxicity. The present study provides an alternative approach for the assessment of other types of PAs for which in vivo genotoxic data are not available, and definition of in vivo REP values, thereby contributing to the reduction, refinement, and replacement of animal testing.

Acknowledgements This work was funded by a grant from the China Scholarship Council to Lu Chen (Grant No. 201508650023).

\section{Compliance with ethical standards}

Conflict of interest The authors declare that they have no conflict of interest.

Open Access This article is distributed under the terms of the Creative Commons Attribution 4.0 International License (http://creativeco mmons.org/licenses/by/4.0/), which permits unrestricted use, distribution, and reproduction in any medium, provided you give appropriate credit to the original author(s) and the source, provide a link to the Creative Commons license, and indicate if changes were made.

\section{References}

Allemang A, Mahony C, Lester C, Pfuhler S (2018) Relative potency of fifteen pyrrolizidine alkaloids to induce DNA damage as measured by micronucleus induction in HepaRG human liver cells. Food Chem Toxicol 121:72-81

Aninat C, Piton A, Glaise D et al (2006) Expression of cytochromes $\mathrm{P} 450$, conjugating enzymes and nuclear receptors in human hepatoma HepaRG cells. Drug Metab Dispos 34(1):75-83

Audebert M, Riu A, Jacques C et al (2010) Use of the $\gamma \mathrm{H} 2 \mathrm{AX}$ assay for assessing the genotoxicity of polycyclic aromatic hydrocarbons in human cell lines. Toxicol Lett 199(2):182-192

Audebert M, Zeman F, Beaudoin R, Pery A, Cravedi J-P (2012) Comparative potency approach based on $\mathrm{H} 2 \mathrm{AX}$ assay for estimating the genotoxicity of polycyclic aromatic hydrocarbons. Toxicol Appl Pharmacol 260(1):58-64

BfR (2013) Pyrrolizidine alkaloids in herbal teas and teas. BfR Opinion No. $018 / 2013$ of 5 July 2013

Bodi D, Ronczka S, Gottschalk C et al (2014) Determination of pyrrolizidine alkaloids in tea, herbal drugs and honey. Food Addit Contam Part A 31(11):1886-1895

Bonner WM, Redon CE, Dickey JS et al (2008) $\gamma \mathrm{H} 2 \mathrm{AX}$ and cancer. Nat Rev Cancer 8(12):957

Candrian U, Lüthy J, Graf U, Schlatter C (1984) Mutagenic activity of the pyrrolizidine alkaloids seneciphylline and senkirkine in Drosophila and their transfer into rat milk. Food Chem Toxicol 22(3):223-225

Chan P, Mahler J, Bucher J, Travlos G, Reid J (1994) Toxicity and carcinogenicity of riddelliine following 13 weeks of treatment to rats and mice. Toxicon 32(8):891-908 
Chen T, Mei N, Fu PP (2010) Genotoxicity of pyrrolizidine alkaloids. J Appl Toxicol 30(3):183-196

Chen L, Ning J, Louisse J, Wesseling S, Rietjens IM (2018) Use of physiologically based kinetic modelling-facilitated reverse dosimetry to convert in vitro cytotoxicity data to predicted in vivo liver toxicity of lasiocarpine and riddelliine in rat. Food Chem Toxicol 116:216-226

Chou MW, Wang YP, Yan J et al (2003) Riddelliine N-oxide is a phytochemical and mammalian metabolite with genotoxic activity that is comparable to the parent pyrrolizidine alkaloid riddelliine. Toxicol Lett 145(3):239-247

Chou MW, Yan J, Nichols J et al (2004) Correlation of DNA adduct formation and riddelliine-induced liver tumorigenesis in F344 rats and B6C3F1 mice. Cancer Lett 207(1):119-125

Clarke JJ, Lawlor TE, Madraymootoo W et al (2012) Summary of in vitro genetic toxicology assay results: expected and unexpected effects of recent study design modifications. Environ Mol Mutagen 53(8):631-635

Creeper J, Mitchell A, Jubb T, Colegate S (1999) Pyrrolizidine alkaloid poisoning of horses grazing a native heliotrope (Heliotropium ovalifolium). Aust Vet J 77(6):401-402

Daston GP, Chapin RE, Scialli AR, Piersma AH, Carney EW, Rogers JM, Friedman JM (2010) A different approach to validating screening assays for developmental toxicity. Birth Defects Res B Dev Reprod Toxicol 89(6):526-530

Edgar JA, Molyneux RJ, Colegate SM (2014) Pyrrolizidine alkaloids: potential role in the etiology of cancers, pulmonary hypertension, congenital anomalies, and liver disease. Chem Res Toxicol 28(1):4-20

EFSA (2011) Scientific opinion on pyrrolizidine alkaloids in food and feed. EFSA Journal 9:2406

EFSA (2016) Dietary exposure assessment to pyrrolizidine alkaloids in the European population. EFSA J 14(8):e04572

EFSA (2017) Risks for human health related to the presence of pyrrolizidine alkaloids in honey, tea, herbal infusions and food supplements. EFSA J 15(7):e04908

Fu P, Chou M, Xia Q et al (2001) Genotoxic pyrrolizidine alkaloids and pyrrolizidine alkaloid N-oxides mechanisms leading to DNA adduct formation and tumorigenicity. J Environ Sci Health C 19(2):353-385

Fu PP, Xia Q, Lin G, Chou MW (2004) Pyrrolizidine alkaloids-genotoxicity, metabolism enzymes, metabolic activation, and mechanisms. Drug Metab Rev 36(1):1-55

Fu PP, Xia Q, Chou MW, Lin G (2007) Detection, hepatotoxicity, and tumorigenicity of pyrrolizidine alkaloids in Chinese herbal plants and herbal dietary supplements. J Food Drug Anal 15(4):400-415

Fu PP, Chou MW, Churchwell M et al (2010) High-performance liquid chromatography electrospray ionization tandem mass spectrometry for the detection and quantitation of pyrrolizidine alkaloidderived DNA adducts in vitro and in vivo. Chem Res Toxicol 23(3):637-652

Gerets HHJ, Tilmant K, Gerin B, Chanteux H, Depelchin BO, Dhalluin S, Atienzar FA (2012) Characterization of primary human hepatocytes, HepG2 cells, and HepaRG cells at the mRNA level and CYP activity in response to inducers and their predictivity for the detection of human hepatotoxins. Cell Biol Toxicol 28(2):69-87

Graillot V, Takakura N, Hegarat LL, Fessard V, Audebert M, Cravedi JP (2012) Genotoxicity of pesticide mixtures present in the diet of the French population. Environ Mol Mutagen 53(3):173-184

Groothuis FA, Heringa MB, Nicol B, Hermens JL, Blaauboer BJ, Kramer NI (2015) Dose metric considerations in in vitro assays to improve quantitative in vitro-in vivo dose extrapolations. Toxicology 332:30-40

Guillouzo A, Corlu A, Aninat C, Glaise D, Morel F, Guguen-Guillouzo C (2007) The human hepatoma HepaRG cells: a highly differentiated model for studies of liver metabolism and toxicity of xenobiotics. Chem Biol Interact 168(1):66-73

Hessel S, Gottschalk C, Schumann D, These A, Preiss Weigert A, Lampen A (2014) Structure-activity relationship in the passage of different pyrrolizidine alkaloids through the gastrointestinal barrier: ABCB1 excretes heliotrine and echimidine. Mol Nutr Food Res 58(5):995-1004

Hill B, Gaul K, Noble J (1997) Poisoning of feedlot cattle by seeds of Heliotropium europaeum. Aust Vet J 75(5):360-361

Hirono I, Mori H, Culvenor CC (1976) Carcinogenic activity of coltsfoot, Tussilago farfara L. GANN Jpn J Cancer Res 67(1):125-129

Hirono I, Haga M, Fujii M et al (1979) Induction of hepatic tumors in rats by senkirkine and symphytine. J Natl Cancer Inst 63(2):469-472

Hoeijmakers JH (2009) DNA damage, aging, and cancer. N Engl J Med 361(15):1475-1485

Imreh G, Norberg HV, Imreh S, Zhivotovsky B (2011) Chromosomal breaks during mitotic catastrophe trigger $\gamma \mathrm{H} 2 \mathrm{AX}-\mathrm{ATM}-\mathrm{p} 53-$ mediated apoptosis. J Cell Sci 124(17):2951-2963

Kempf M, Reinhard A, Beuerle T (2010) Pyrrolizidine alkaloids (PAs) in honey and pollen-legal regulation of PA levels in food and animal feed required. Mol Nutr Food Res 54(1):158-168

Khoury L, Zalko D, Audebert M (2013) Validation of high-throughput genotoxicity assay screening using $\gamma \mathrm{H} 2 \mathrm{AX}$ in-cell western assay on HepG2 cells. Environ Mol Mutagen 54(9):737-746

Kim HY, Stermitz FR, Coulombe RA Jr (1995) Pyrrolizidine alkaloid-induced DNA-protein cross-links. Carcinogenesis 16(11):2691-2697

Kopp B, Zalko D, Audebert M (2018) Genotoxicity of 11 heavy metals detected as food contaminants in two human cell lines. Environ Mol Mutagen 59(3):202-210

Kuhara K, Takanashi H, Hirono I, Furuya T, Asada Y (1980) Carcinogenic activity of clivorine, a pyrrolizidine alkaloid isolated from Ligularia dentata. Cancer Lett 10(2):117-122

Kvist AJ, Kanebratt KP, Walentinsson A, Palmgren H, O'Hara M, Bjorkbom A, Andersson LC, Ahlqvist M, Andersson TB (2018) Critical differences in drug metabolic properties of human hepatic cellular models, including primary human hepatocytes, stem cell derived hepatocytes, and hepatoma cell lines. Biochem Pharmacol 155:124-140

Li N, Xia QS, Ruan JQ, Fu PP, Lin G (2011) Hepatotoxicity and tumorigenicity induced by metabolic activation of pyrrolizidine alkaloids in herbs. Curr Drug Metab 12(9):823-834

Lin G, Cui YY, Liu XQ (2003) Gender differences in microsomal metabolic activation of hepatotoxic clivorine in rat. Chem Res Toxicol 16(6):768-774

Lin G, Wang JY, Li N et al (2011) Hepatic sinusoidal obstruction syndrome associated with consumption of Gynura segetum. J Hepatol 54(4):666-673

Louisse J, Rijkers D, Stoopen G, Holleboom WJ, Delagrange M, Molthof E, Mulder PP, Hoogenboom RL, Audebert M, Peijnenburg AA (2019) Determination of genotoxic potencies of pyrrolizidine alkaloids in HepaRG cells using the $\gamma \mathrm{H} 2 \mathrm{AX}$ assay. Food Chem Toxicol 131:110532

Lübberstedt M, Muller-Vieira U, Mayer M, Biemel KM, Knospel F, Knobeloch D, Nussler AK, Gerlach JC, Zeilinger K (2011) HepaRG human hepatic cell line utility as a surrogate for primary human hepatocytes in drug metabolism assessment in vitro. $\mathbf{J}$ Pharmacol Toxicol 63(1):59-68

Mathon C, Edder P, Bieri S, Christen P (2014) Survey of pyrrolizidine alkaloids in teas and herbal teas on the Swiss market using HPLCMS/MS. Anal Bioanal Chem 406(28):7345-7354

Mattocks A (1986) Chemistry and toxicology of pyrrolizidine alkaloids. Academic, London, p 393

Miranda CL, Reed RL, Guengerich FP, Buhler DR (1991) Role of cytochrome P450IIIA4 in the metabolism of the 
pyrrolizidine alkaloid senecionine in human liver. Carcinogenesis 12(3):515-519

Molyneux R, Gardner D, Colegate S, Edgar J (2011) Pyrrolizidine alkaloid toxicity in livestock: a paradigm for human poisoning? Food Addit Contam Part A 28(3):293-307

Mori H, Sugie S, Yoshimi N, Asada Y, Furuya T, Williams GM (1985) Genotoxicity of a variety of pyrrolizidine alkaloids in the hepatocyte primary culture-DNA repair test using rat, mouse, and hamster hepatocytes. Cancer Res 45(7):3125-3129

Ning J, Chen L, Strikwold M, Louisse J, Wesseling S, Rietjens IM (2019) Use of an in vitro-in silico testing strategy to predict interspecies and inter-ethnic human differences in liver toxicity of the pyrrolizidine alkaloids lasiocarpine and riddelliine. Arch Toxicol 93(3):801-818

NTP (1978) Bioassay of lasiocarpine for possible carcinogenicity. Natl Cancer Inst Carcinog Tech Rep Ser 39:1

NTP (2003) Toxicology and carcinogenesis studies of riddelliine (CAS No. 23246-96-0) in F344/N rats and B6C3F1 mice (gavage studies). Natl Toxicol Progr Tech Rep Ser 508:1

O'donovan M (2012) A critique of methods to measure cytotoxicity in mammalian cell genotoxicity assays. Mutagenesis 27(6):615-621

Prakash AS, Pereira TN, Reilly PE, Seawright AA (1999) Pyrrolizidine alkaloids in human diet. Mutat Res Genet Toxicol Environ Mutagen 443(1):53-67

Raquet N, Schrenk D (2014) Application of the equivalency factor concept to the phototoxicity and -genotoxicity of furocoumarin mixtures. Food Chem Toxicol 68:257-266

Roeder E (2000) Medicinal plants in China containing pyrrolizidine alkaloids. Pharmazie 55:I0

Sedelnikova OA, Redon CE, Dickey JS, Nakamura AJ, Georgakilas AG, Bonner WM (2010) Role of oxidatively induced DNA lesions in human pathogenesis. Mutat Res Rev Mutat Res 704(1-3):152-159

Shumaker R, Robertson K, Hsu I, Allen J (1976) Neoplastic transformation in tissues of rats exposed to monocrotaline or dehydroretronecine. J Natl Cancer Inst 56(4):787-790

Singh SK, Wang M, Staudt C, Iliakis G (2011) Post-irradiation chemical processing of DNA damage generates double-strand breaks in cells already engaged in repair. Nucleic Acids Res 39(19):8416-8429

Suzuki G, Sato K, Isobe T, Takigami H, Brouwer A, Nakayama K (2015) Detection of glucocorticoid receptor agonists in effluents from sewage treatment plants in Japan. Sci Total Environ 527-528:328-334
Takanashi H, Umeda M, Hirono I (1980) Chromosomal aberrations and mutation in cultured mammalian cells induced by pyrrolizidine alkaloids. Mutation Res/Genet Toxicol 78(1):67-77

Theumer M, Henneb Y, Khoury L et al (2018) Genotoxicity of aflatoxins and their precursors in human cells. Toxicol Lett 287:100-107

Turpeinen M, Tolonen A, Chesne C, Guillouzo A, Uusitalo J, Pelkonen O (2009) Functional expression, inhibition and induction of CYP enzymes in HepaRG cells. Toxicol In Vitro 23(4):748-753

Uhl M, Helma C, Knasmüller S (2000) Evaluation of the single cell gel electrophoresis assay with human hepatoma (Hep G2) cells. Mutat Res Genet Toxicol Environ Mutagen 468(2):213-225

Van den Berg M, Birnbaum LS, Denison M et al (2006) The 2005 world health organization reevaluation of human and mammalian toxic equivalency factors for dioxins and dioxin-like compounds. Toxicol Sci 93(2):223-241

Wang YP, Fu PP, Chou MW (2005a) Metabolic activation of the tumorigenic pyrrolizidine alkaloid, retrorsine, leading to DNA adduct formation in vivo. Int J Environ Res Publ Health 2(1):74-79

Wang YP, Yan J, Beger RD, Fu PP, Chou MW (2005b) Metabolic activation of the tumorigenic pyrrolizidine alkaloid, monocrotaline, leading to DNA adduct formation in vivo. Cancer Lett 226(1):27-35

Wiedenfeld H (2011) Plants containing pyrrolizidine alkaloids: toxicity and problems. Food Addit Contam Part A 28(3):282-292

Xia Q, Chou MW, Edgar JA, Doerge DR, Fu PP (2006) Formation of DHP-derived DNA adducts from metabolic activation of the prototype heliotridine-type pyrrolizidine alkaloid, lasiocarpine. Cancer Lett 231(1):138-145

Xia Q, Zhao Y, Von Tungeln LS et al (2013) Pyrrolizidine alkaloidderived DNA adducts as a common biological biomarker of pyrrolizidine alkaloid-induced tumorigenicity. Chem Res Toxicol 26(9):1384-1396

Yan J, Nichols J, Yang YC, Fu PP, Chou MW (2002) Detection of riddelliine-derived DNA adducts in blood of rats fed riddelliine. Int J Mol Sci 3(9):1019-1026

Publisher's Note Springer Nature remains neutral with regard to jurisdictional claims in published maps and institutional affiliations. 